\title{
Gene-environment interaction for body size and larval density in Drosophila melanogaster: an investigation of effects on development time, thorax length and adult sex ratio
}

\author{
MAURO SANTOS $\dagger$, KEVIN FOWLER $\ddagger \&$ LINDA PARTRIDGE $*^{*}$ \\ Institute of Cell, Animal and Population Biology, University of Edinburgh, West Mains Road, Edinburgh EH9 3JT, UK
}

\begin{abstract}
We measured the effect of larval density on thorax length, development time, sex ratio and a measure of total fitness, using strains of Drosophila melanogaster artificially selected for increased thorax length, control lines otherwise cultured in an identical way, and the base stock from which the lines had been derived. We used the addition experimental design (Mather \& Caligari, 1981). No genotype-environment interaction was observed when comparing the reduction in thorax length of 'large' and 'control' lines with increasing larval density for any culture series, i.e. rank ordering of genotypes and additive genetic variances remained the same in all the environments tested. In contrast, the reduction in thorax length for the base stock as density increased was proportionally smaller than that of the 'large' and 'control' lines. Development time increased more rapidly with larval density in the 'large' lines than in the 'controls' or base stock. Sex ratio was unaffected by larval density but thorax length and the development time of females were more affected than those of males by increasing larval density. The estimate of total fitness showed clear evidence of gene-environment interaction for the effect of body size on fitness, with genetically large individuals at an increasing disadvantage with increasing larval density.
\end{abstract}

Keywords: body size, development time, Drosophila melanogaster, gene-environment interaction, larval density, sex ratio.

\section{Introduction}

Body size has major effects on fitness in many organisms including Drosophila. In general, adult fitness increases with body size in adult Drosophila, with female fertility, male mating success and longevity all affected (e.g. Alpatov, 1929; Robertson, 1957; Tantawy \& Vetukhiv, 1960; Tantawy \& Rakha, 1964; Partridge \& Farquhar, 1981, 1983; Partridge et al., 1987a, b; Wilkinson, 1987; Markow, 1988; Santos et al., 1988, 1992b; Taylor \& Kekic, 1988). In contrast, artificial selection for increased thorax length in $D$. melanogaster has been shown to cause a correlated

\section{*Correspondence.}

†Permanent address: Departamento de Genetica y Microbiologia, Universidad Automona de Barcelona, 08193 Bellaterra, Barcelona, Spain.

$\Varangle$ Present address: Department of Genetics, University College London, Wolfson House, 4 Stephenson Way, London NW1 2HE, UK. drop in larval survival, at least at higher larval densities (Santos et al., 1992a; Partridge \& Fowler, 1993). Observed body size in adult Drosophila may therefore represent a compromise between conflicting effects of genetic variation for the trait on the fitness of larvae and adults.

The aim of this study was to examine the interaction between the effects of artificial selection for increased thorax length and changes in larval density. We previously showed that pre-adult survival of 'large' selected lines decreased disproportionately to that of 'control' lines and of the base stock as larval density was increased (Santos et al., 1992a; Partridge \& Fowler, 1993). We here test for additional effects of gene-environment interaction on pre-adult development time, adult thorax length, adult sex ratio and an estimate of total fitness.

Development time can affect fitness in Drosophila both by its effect on pre-adult mortality and by deter- 
mining the age of first breeding. In many parts of its geographical range, D. melanogaster breeds mainly during periods of population expansion, when early breeding and hence rapid larval development are favoured (Cole, 1954; Lewontin, 1965). Any interaction for development time between genetic variation for body size and larval density would therefore result in spatially or temporally variable selection on body size.

We also examined the effect of increasing larval density on the thorax length of 'large', 'control' and base stock adults. The selection lines used in the experiment were selected and initially measured under conditions of relaxed larval competition (Partridge \& Fowler, 1993). Any interaction between selection regime and larval density for thorax length would be mirrored in effects on adult fitness because of the strong association with body size.

Finally, we also examined the interaction between genetic variation for body size and larval density on sex ratio. D. melanogaster females are larger than males and since 'large' selected lines suffered more of an increase in larval mortality than did 'controls' as larval density increased (Santos et al., 1992a; Partridge \& Fowler, 1993) the same might be expected to be true for females as compared with males.

In this experiment we altered larval density using the so-called addition design (Mather \& Caligari, 1981). In contrast with the substitution or replacement design where density is kept constant in mixed cultures (de Wit, 1960; Mather \& Caligari, 1981), density varies through the experimental series in the addition design. Although the effect of competition would probably be similar in both cases (de Miranda \& Eggleston, 1987), the addition design conforms with the natural situation, as the crowding conditions experienced by Drosophila larvae are different among individual breeding sites (e.g. Grimaldi \& Jaenike, 1984). There is increasing evidence that intra- and interspecific competition are important ingredients of larval life under field conditions (Atkinson, 1979, 1985; Grimaldi \& Jaenike, 1984; Prout \& Barker, 1989), so that laboratory studies of the effects of larval density are important for understanding ecological variation in selection for body size in Drosophila.

Gene-environment interaction for the effect of a trait on fitness could result in both local population differentiation and the maintenance of genetic variation for the trait. Gillespie \& Turelli (1989) have stressed the importance of genotype-environment interaction for maintaining genetic variation in quantitative characters which, in turn, has an important bearing on estimates of natural heritabilities of body size in Drosophila because they involve the trait's expression across environments (Riska et al., 1989).

\section{Materials and methods}

Five different strains of Drosophila melanogaster, three wild-type and two eye colour mutants, were used (see Santos et al. (1992a) for details). Briefly, two of the wild-type strains were, respectively, the poly-hybrid of four laboratory lines selected for increasing thorax length (L) and the poly-hybrid of four lines that served as the controls for the selection programme (C) (see Wilkinson et al. (1990) and Partridge \& Fowler (1993) for further details of the selection lines). All these lines were derived about 10 years ago from the outbred base stock Dahomey (D), the third wild-type strain used. This stock was also used to provide the eye mutant strains sparkling poliert (spapol; 4) and scarlet (st; 3-44.0).

Competitive interactions were analysed by comparison of the linear regression slopes of mono- and duocultures following the addition design of Mather \& Caligari (1981). Competition took place in 2.5 $\mathrm{cm} \times 7.5 \mathrm{~cm}$ glass vials, each containing $3 \mathrm{ml} 2.5$ per cent agar gel as a non-nutritive base and $3 \mathrm{ml}$ of food medium added after the agar cooled (Santos et al., 1992a). Competition was started by seeding a reference number of 30 first instar larvae (age range of up to $2 \mathrm{~h}$ after hatching) of the indicator strain and then adding 30,60, 120 and 210 larvae of the indicator (monocultures) or eye mutant (duocultures) strains. The competitive ability of the mutant strains had been estimated previously (Santos et al., 1992a).

For 10 days following the emergence of the first adults, flies were collected twice daily $(08.00-11.00 \mathrm{~h}$ and $19.00-23.00 \mathrm{~h}$ ) and kept in small vials at $-20^{\circ} \mathrm{C}$ for analysis during the month after emergence. The mean thorax length, larva-adult development time and sex ratio of the emerging wild-type adults were measured. Adult thorax length was measured to the nearest $0.02 \mathrm{~mm}$ with a binocular microscope fitted with an ocular micrometer, from the anterior margin of the thorax to the posterior tip of the scutellum, as viewed from the side. Up to three females and three males per collection were measured for thorax length. The average thorax lengths of the females and males from a culture were calculated weighting the mean of each collection by the number of flies of each sex scored in that collection. Larva-to-adult development time was estimated as the average number of $h$ from culture initiation to adult emergence, where all females and males counted at a particular scoring were taken as having emerged at the midpoint in time between that scoring and the previous one.

Full statistical details of the analyses are given in Santos et al. (1992a). Throughout the whole experiment, no more than six entries of 312 were lost. In one vial, no wild-type flies were raised and only males or 
females were scored in five vials. In all cases but two (13 per cent), the linear model was found to be adequate.

\section{Results}

\section{Thorax length}

Mean thorax length for females and males at each density and culture are set out in Table 1 . For statistical analyses, each observed value was converted to natural logarithms to make the variances independent of the mean and to remove or reduce non-additive interactions. The male and female thorax lengths were subjected to analyses of variance to test whether a linear model was an adequate fit. The residual variation around the regression lines was higher than the error variance in only one of six comparisons (and that was significant only at the 5 per cent level) so that it is reasonable to assume a linear response between thorax length and density.

The estimates of the parameters obtained for the three wild-type strains are given in Tables 2 and 3. It is clear that increasing the number of larvae significantly reduced the thorax length of the adults and the gradient

Table 1 Thorax length (in mm) of wild-type females (above) and males (below)

\begin{tabular}{lccccc}
\hline & \multicolumn{5}{c}{ Density } \\
\cline { 2 - 5 } Culture & \multirow{2}{*}{30} & 60 & 90 & 150 & 240 \\
\hline L/- & 1.161 & 1.150 & 1.116 & 1.082 & 1.064 \\
& 0.966 & 0.996 & 0.954 & 0.947 & 0.928 \\
L/spa & & $1.126^{\dagger}$ & 1.068 & 0.995 & $0.957^{\dagger}$ \\
& & 0.986 & 0.931 & $0.867^{\dagger}$ & 0.833 \\
L/st & & 1.103 & 1.067 & $0.966^{\dagger}$ & $0.884^{\dagger}$ \\
& & 0.952 & $0.992^{\dagger}$ & $0.833^{\dagger}$ & $0.787^{\ddagger}$ \\
C/- & 1.061 & 1.039 & 0.996 & 0.992 & 0.961 \\
& 0.919 & 0.910 & 0.881 & 0.875 & 0.846 \\
C/spa & & 0.999 & 1.006 & $0.924^{\dagger}$ & 0.844 \\
& & 0.889 & 0.873 & 0.823 & 0.760 \\
C/st & & 1.008 & 0.943 & 0.889 & 0.817 \\
& & $0.882^{\dagger}$ & 0.843 & 0.782 & 0.743 \\
D/- & 1.092 & 1.041 & 1.034 & 0.984 & 0.941 \\
& 0.942 & 0.902 & 0.907 & 0.866 & 0.838 \\
$\mathrm{D} /$ spa & & 1.058 & 1.033 & 0.989 & 0.957 \\
& & 0.929 & 0.906 & 0.877 & 0.850 \\
$\mathrm{D} /$ st & & 1.034 & 0.988 & 0.934 & 0.861 \\
& & 0.901 & 0.879 & 0.837 & 0.777 \\
\hline
\end{tabular}

Each figure is the average of eight replicates.

$\$ 7$ replicates.

¥6 replicates. of the female regression for all culture series and strains was greater than that of males. This means that as density increased from 30 to 240 larvae, the sex difference in thorax length decreased, a result consistent with other data for Drosophila (Ashburner \& Thompson, 1978).

As shown in Santos et al. (1992a), comparisons between the various partial regression coefficients for the three wild-type strains allow us to infer the effects that selection for increasing thorax length and/or past selective experience in uncrowded conditions has had on thorax length.

Several things are evident from the results. Firstly, inter-genotypic effects on thorax length ( $\hat{\beta}_{\mathrm{d}}$ values) were stronger than intra-genotypic ones ( $\hat{\beta}_{\mathrm{m}}$ values $)$ for the $\mathrm{L}$ and $\mathrm{C}$ strains and no differences between monoand duocultures with spapol were detected for the D strain. In all cases, st larvae were the strongest intergenotypic competitors. Secondly, the outcome of duocultures could not be predicted from that of monocultures as intra-genotypic effects for the Dahomey strain were stronger than those observed for $\mathrm{L}$ and $\mathrm{C}$ strains. Thirdly, at the optimum density of 30 larvae per vial, flies from the $\mathrm{L}$ strain were larger than flies from $\mathrm{C}$ and $\mathrm{D}$. This was an obvious expectation as $\mathrm{L}$ flies were derived from four lines artificially selected for increasing thorax length for more than 200 generations. On the other hand, the significantly larger body size of flies from the Dahomey base stock compared

Table 2 Estimates of the parameters ( \pm S.E.) for the variable female thorax length (in $\ln \mathrm{mm}$ )

\begin{tabular}{lrccc}
\hline Strain & \multicolumn{1}{c}{$\beta_{0}$} & $\beta_{\mathrm{m}}$ & $\beta_{\mathrm{d} s p a}$ & $\beta_{\mathrm{d} s t}$ \\
\hline $\mathbf{L}$ & 0.136731 & -0.000389 & -0.000966 & -0.001288 \\
& \pm 0.006830 & \pm 0.000073 & \pm 0.000076 & \pm 0.000080 \\
$\mathrm{C}$ & 0.039898 & -0.000406 & -0.000981 & -0.001219 \\
& \pm 0.005642 & \pm 0.000061 & \pm 0.000062 & \pm 0.000061 \\
$\mathrm{D}$ & 0.066442 & -0.000629 & -0.000556 & -0.001070 \\
& \pm 0.005205 & \pm 0.000056 & \pm 0.000056 & \pm 0.000056 \\
\hline
\end{tabular}

Table 3 Estimates of the parameters ( \pm S.E.) for the variable male thorax length (in $\ln \mathrm{mm}$ )

\begin{tabular}{lcccc}
\hline Strain & $\beta_{0}$ & $\beta_{\mathrm{m}}$ & $\beta_{\mathrm{dspa}}$ & $\beta_{\mathrm{d} s t}$ \\
\hline L & -0.011318 & -0.000330 & -0.000885 & -0.001193 \\
& \pm 0.007671 & \pm 0.000083 & \pm 0.000083 & \pm 0.000090 \\
$\mathrm{C}$ & -0.094207 & -0.000358 & -0.000849 & -0.001058 \\
& \pm 0.006311 & \pm 0.000068 & \pm 0.000068 & \pm 0.000068 \\
$\mathrm{D}$ & -0.072930 & -0.000522 & -0.000439 & $-\mathbf{0 . 0 0 0 8 6 9}$ \\
& \pm 0.004761 & \pm 0.000052 & \pm 0.000052 & \pm 0.000052
\end{tabular}


with that of the $\mathrm{C}$ flies suggests that some other effect of the selection regime reduced thorax length. Fourthly, changes in thorax length with changes in density were the same for the $\mathrm{L}$ and $\mathrm{C}$ strains in all culture series. This means that no genotype-environment interaction was present and differences in body size between genetically large flies and their corresponding controls remained constant through densities. However, this was not true for $\mathrm{L}$ vs. D and $\mathrm{C}$ vs. D comparisons, where the reduction in body size with increasing larval density was greater for the selection lines.

\section{Larva-to-adult developmental period}

The mean development times for each culture series are shown in Table 4. In general, the three indicator genotypes exhibited the typical pattern of D. melanogaster, with females emerging earlier than males. There was a positive correlation between means and variances as density increased from 30 to 240 larvae for all indicator genotypes. Therefore, all values were converted to common logarithms for statistical analyses.

The male and female development times were subjected to analyses of variance that tested the goodness

Table 4 Larva-adult development time (in h) of wild-type females (above) and males (below)

\begin{tabular}{|c|c|c|c|c|c|}
\hline \multirow[b]{2}{*}{ Culture } & \multicolumn{5}{|c|}{ Density } \\
\hline & 30 & 60 & 90 & 150 & 240 \\
\hline \multirow[t]{2}{*}{ L/- } & 235 & 239 & 244 & 252 & 259 \\
\hline & 239 & 243 & 248 & 255 & 264 \\
\hline \multirow[t]{2}{*}{$\mathrm{L} / s p a$} & & $236^{\dagger}$ & 250 & 267 & $315^{\dagger}$ \\
\hline & & 242 & 249 & $268^{\dagger}$ & 305 \\
\hline \multirow{2}{*}{$\mathrm{L} / s t$} & & 252 & 265 & $341^{\dagger}$ & $341^{\ddagger}$ \\
\hline & & 252 & $261^{\dagger}$ & $334^{\dagger}$ & $345^{\ddagger}$ \\
\hline \multirow{2}{*}{$\mathrm{Cl}-$} & 221 & 229 & 242 & 237 & 251 \\
\hline & 224 & 230 & 247 & 240 & 259 \\
\hline \multirow[t]{2}{*}{$\mathrm{C} / \mathrm{spa}$} & & 244 & 231 & $256^{\dagger}$ & 277 \\
\hline & & 246 & 238 & 250 & 280 \\
\hline \multirow[t]{2}{*}{$\mathrm{C} / s t$} & & 234 & 251 & 271 & 309 \\
\hline & & $237^{\dagger}$ & 257 & 285 & 303 \\
\hline \multirow[t]{2}{*}{$\mathrm{D} /-$} & 216 & 223 & 227 & 250 & 271 \\
\hline & 225 & 227 & 231 & 252 & 272 \\
\hline \multirow[t]{2}{*}{$\mathrm{D} / s p a$} & & 221 & 225 & 231 & 255 \\
\hline & & 225 & 232 & 236 & 256 \\
\hline \multirow[t]{2}{*}{$\mathrm{D} / s t$} & & 228 & 236 & 262 & 300 \\
\hline & & 233 & 235 & 268 & 307 \\
\hline
\end{tabular}

Each figure is the average of eight replicates.

$\dagger 7$ replicates.

$\ddagger 6$ replicates. of fit of a linear model. The residual variation was higher than the error variance in only one of six comparisons (and in that case was significant only at the 5 per cent level) so that it was reasonable to assume a linear response between development time and density. The parameter estimates are set out in Tables 5 and 6 . It is obvious from these tables that increasing the number of larvae significantly increased development time and, as density increased, the development time of females was, in general, proportionally increased more than that of males.

There are several conclusions from a comparison of the $\beta$ values. At the reference density of 30 larvae, the $\mathrm{L}$ strain had the longest estimated development time whereas the Dahomey stock had the shortest (Tables 5 and 6). The difference between $\mathrm{L}$ and $\mathrm{C}$ was not statistically significant for males, although the magnitude of the difference was comparable for the sexes (Table 4). In addition, from the mean values observed in Table 4, it is clear that differences between C and D at the reference density were slight, so that statistical significance detected when comparing the $\hat{\beta}_{0}$ estimates may be somewhat surprising. Least squares estimation minimizes the residual variation in the whole data set and some disparity between observed and estimated differences at the y-intercept (i.e. at the reference density) may appear. Hence, the conclusion that $L$ strain flies had a longer developmental period than their corresponding controls seems sound but some caution is needed when comparing $C$ and $D$ strains.

Table 5 Estimates of the parameters ( \pm S.E.) for the variable female development time (in $\log \mathrm{h}$ )

\begin{tabular}{lrrrr}
\hline Strain & \multicolumn{1}{c}{$\beta_{0}$} & \multicolumn{1}{c}{$\beta_{\mathrm{m}}$} & \multicolumn{1}{c}{$\beta_{\mathrm{d} s p a}$} & \multicolumn{1}{c}{$\beta_{\mathrm{d} s t}$} \\
\hline $\mathrm{L}$ & 2.370436 & 0.000212 & 0.000550 & 0.000917 \\
& \pm 0.005783 & \pm 0.000062 & \pm 0.000064 & \pm 0.000068 \\
$\mathrm{C}$ & 2.354302 & 0.000217 & 0.000412 & 0.000641 \\
& \pm 0.005395 & \pm 0.000058 & \pm 0.000059 & \pm 0.000058 \\
$\mathrm{D}$ & 2.333174 & 0.000480 & 0.000319 & 0.000685 \\
& \pm 0.003883 & \pm 0.000042 & \pm 0.000042 & \pm 0.000042 \\
\hline
\end{tabular}

Table 6 Estimates of the parameters $( \pm$ S.E.) for the variable male development time (in $\log \mathrm{h}$ )

\begin{tabular}{lrrrr}
\hline Strain & \multicolumn{1}{c}{$\beta_{0}$} & \multicolumn{1}{c}{$\beta_{\mathrm{m}}$} & \multicolumn{1}{c}{$\beta_{\mathrm{d} s p a}$} & \multicolumn{1}{c}{$\beta_{\mathrm{d} s t}$} \\
\hline $\mathrm{L}$ & 2.375174 & 0.000230 & 0.000478 & 0.000881 \\
& \pm 0.005364 & \pm 0.000058 & \pm 0.000058 & \pm 0.000063 \\
$\mathrm{C}$ & 2.362325 & 0.000225 & 0.000371 & 0.000612 \\
& \pm 0.005353 & \pm 0.000058 & \pm 0.000058 & \pm 0.000058 \\
$\mathrm{D}$ & 2.343489 & 0.000434 & 0.000284 & 0.000666 \\
& \pm 0.005301 & \pm 0.000058 & \pm 0.000058 & \pm 0.000058
\end{tabular}


Within indicator genotypes, the performance of the characters thorax length and development time with density for mono- and duocultures were very similar. The main disagreement arose from the duoculture slope comparisons where a difference between elevations for the $\mathbf{L}$ and $\mathrm{C}$ strains was observed. Increasing the number of mutant larvae in the duoculture series had a major effect on the development time of the large flies, which increased proportionally more than that of the control flies. The difference was highly significant against the strongest competitor, i.e. the st larvae. Hence, a clear indication of genotype-environment interaction is present. On the other hand, no difference was detected this time between the $\mathrm{C}$ and $\mathrm{D}$ strains.

\section{Sex ratio}

This was calculated for each indicator genotype at each density of mono- and duocultures as the proportion of males, and deviations from the $1: 1$ sex ratio were tested against expected binomial frequencies. Only one out of 39 cases ( 2.6 per cent) showed a significant departure from the expected sex ratio and no relationship was observed between the proportion of males and density in any strain.

\section{Density-related fitnesses}

A comparison of overall performance for genetically large flies and their corresponding controls may be assessed by means of the index: (viability $\times$ thorax length)/(development time). As thorax length covaries positively with adult fitness components, this index can be taken as a measure of total fitness.

The relationship between relative fitness for the $\mathrm{L}$ and $\mathrm{C}$ strains against density (using the viability data from Santos et al., 1992a) is shown in Fig. 1. A clear pattern of genotype-environment interaction is observed, with overall performance of genetically large flies being somewhat higher at low densities than that of the $\mathrm{C}$ flies whereas the opposite is true at moderate and high densities.

\section{Discussion}

An important finding was the lack of genotypeenvironment interaction for thorax length when the performances of the $\mathrm{L}$ and $\mathrm{C}$ strains in relation to larval density in duocultures were compared. If an important feature of the larval environment in the field is variation in larval crowding among breeding sites, then extrapolation of our data suggests that rank ordering of genotypes and additive genetic variances in the different environments will be the same. Hence, positive offspring-parent regressions for thorax length would be expected and have indeed been reported (Coyne \& Beecham, 1987). Similar results have been shown for $D$. buzzatii, which shows no substantial genotype-environment interaction for body size (Prout \& Barker, 1989) and positive heritability values from field-caught adults (Prout \& Barker, 1989; Ruiz et al., 1991).

These results can be explained in terms of the traits known to influence the development of adult body size in Drosophila (Bakker, 1959, 1961; Robertson, 1963; Burnet et al., 1977). The larval growth phase can be divided into two parts: a variable period between hatching and the attainment of a critical size at which an irreversible commitment to pupation occurs, followed by a constant period up till pupation. Future adult size will be mainly determined by food availability during the post-critical period. If food is in short supply, growth will be greatly reduced or even stopped and adult body size will decrease to a minimal value (Sang, 1949; Miller, 1964; Caligari, 1980). Aritificial selection for large body size does not change larval
Fig. 1 Relationship between relative fitness and larval density for large $(\mathrm{L})$ and control (C) strains in duocultures with (a) sparkling poliert mutant strain and (b) scarlet mutant strain.
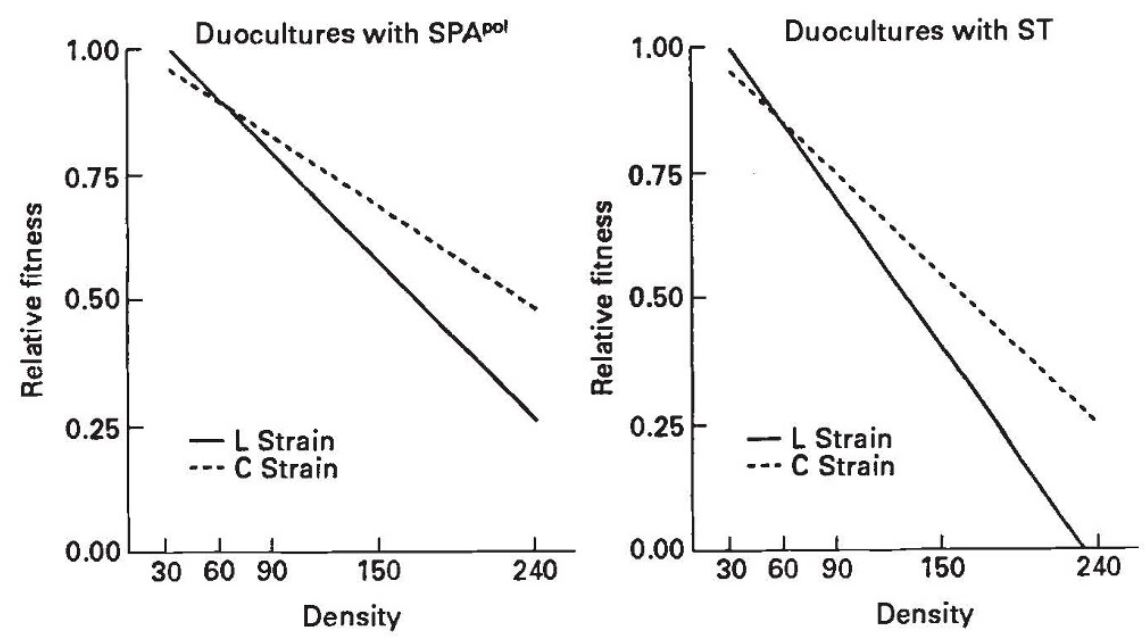
growth rate (Robertson, 1963; L. Partridge, R. E. Langelan, K. Fowler \& V. French, unpublished data), which already appears to be at an evolutionary maximum (Sewell et al., 1975; Burnet et al., 1977) but it appears to result in a later arrival at the critical weight for pupation, implying that this increases with artificial selection for large body size (Robertson, 1963; L. Partridge, R. E. Langelan, K. Fowler \& V. French, unpublished data). Absolute size at the larval critical stage is correlated with that of the adult (Church \& Robertson, 1966). The roughly constant difference in thorax length between large and control lines observed through all culture series therefore probably reflects the prior difference in larval size at the critical stage.

However, this explanation cannot apply to the duoculture series for the Dahomey flies because their thorax length was more resistant to reduction by the effects of increasing larval density. This may have reflected the evolutionary history of the Dahomey stock. It was maintained since collection in 1970 in population cage cultures at their carrying capacity. The stock may have a higher efficiency of food utilization, a higher tolerance of waste products or both. One important consequence of larval crowding, in addition to limiting available food, is the increased concentration of detrimental waste products which can inhibit larval growth (Weisbrot, 1966; Dawood \& Strickberger, 1969; Budnic \& Brncic, 1976; Botella et al., 1985). As both $L$ and $C$ strains came from lines that had a past history of low larval competition, high concentrations of metabolic end products such as urea and uric acid may have had a larger effect on them than on the Dahomey stock. An evolutionary increase in resistance to the effects of larval crowding has been reported in lines cultured at high larval density (Bierbaum et al., 1989).

There was clear evidence that the development time of the large lines was more sensitive than that of the controls to increased larval density. This finding is consistent with increased critical weight in the large lines. If growth rate is slowed to an equal extent in large and control lines by larval crowding, then the development period of the large lines would be extended more. Thus, variation in larval density could be a potent selective force maintaining genetic variation and population differentiation for body size in the field.

Increasing larval density decreased the body size of females more than that of males and also caused a greater increase in development time but did not result in differential pre-adult mortality of the sexes. Female larvae have higher growth rates and higher critical weights than do males but the sexes do not differ in body weight at hatching (L. Partridge, R. E. Langelan, K. Fowler \& V. French, unpublished data). These effects may make the survival rates of the sexes similar at all larval densities. However, this cannot explain the observed effects of larval crowding on females. The pattern could be explained if female growth rate were more affected than that of males but this would be expected to result in higher female than male mortality. However, no consistent deviation from a $1: 1$ sex ratio was observed in the experiment. At present, the different effects on the sexes of larval crowding remain unexplained.

The estimates of total fitness that include effects on larvae and adults suggest that there is gene-environment interaction for the effect of body size on total fitness. With increasing larval density, there is increasing selection against genetically large individuals. The data therefore support the importance both of tradeoffs between the larval and adult period and of gene-environment interaction in relation to larval density as important selective forces on body size in Drosophila.

\section{Acknowledgements}

Financial support from the Direccion General de Investigacion Cientifica y Tecnica (Spain) to M.S., S.E.R.C. (U.K.) to L.P. and The Royal Society (U.K.) to K.F. is gratefully acknowledged.

\section{References}

Alpatov, w. w. 1929. Growth and variation of the larvae of Drosophila melanogaster. J. Exp. Zool., 42, 407-437.

ATKINSON, w. D. 1979. A field investigation of larval competition in domestic Drosophila. J. Anim. Ecol., 48, 91-102.

ATKINSON, W. D. 1985. Coexistence of Australian rain forest Diptera breeding in a fallen fruit. J. Anim. Ecol., 54, 507-518.

ASHBURNER, M. AND THOMPSON, J. N. 1978. The laboratory culture of Drosophila. In: Ashburner, M. and Wright, T. R. F. (eds) The Genetics and Biology of Drosophila, vol. 2a, pp. 1-109. Academic Press, London.

BAKKER, K. 1959. Feeding period, growth and pupation in larvae of Drosophila melanogaster. Ent. Exp. Appl., 2, 171-186.

BAKKER, K. 1961. An analysis of factors which determine success in competition for food among larvae of Drosophila melanogaster. Arch. Neerl. Zool., 14, 200-281.

BIERBAUM, T. J., MUELler, L. D. AND AYAlA, F. J. 1989. Densitydependent evolution of life-history traits in Drosophila melanogaster. Evolution, 43, 382-392.

BOTELLA, L. M., MOYA, A., GONZALEZ, M. C. AND MENSUA, J. L. 1985. Larval stop, delayed development and survival in overcrowded cultures of Drosophila melanogaster. Effect of urea and uric acid. J. Insect Physiol., 31, 179-185.

BUDNIK, M. AND BRNCIC, D. 1976. Effects of larval biotic residues on viability of four species of Drosophila. Evolution, 29, 777-781. 
BURNET, B., SEWELL, D. AND BOS, M. 1977. Genetic analysis of larval feeding behaviour in Drosophila melanogaster. II. Growth relations and competition between selected lines. Genet. Res., 30, 149-161.

CALIGARI, P. D. S. 1980. Competitive interactions in Drosophila melanogaster. I. Monocultures. Heredity, 45, 219-231.

CHURCH, R. B. AND ROBERTSON, F. W. 1966. Biochemical analysis of genetic differences in the growth of Drosophila melanogaster. Genet. Res., 7, 385-407.

COLE, L. C. 1954. The population consequences of life history phenomena. Q. Rev. Biol., 29, 103-137.

COYNE, J. A. AND BEECHAM, E. 1987. Heritability of two morphological characters within and among natural populations of Drosophila melanogaster. Genetics, 117, 727-737.

DAWOOD, M. M. AND STRICKBERGER, M. W. 1969. The effects of larval interaction on viability in Drosophila melanogaster. IIl. Effects of biotic residues. Genetics, 63, 213-220.

DE MIRANDA, J. R. AND EGGLESTON, P. 1987. A comparison of substitution and addition design for the analysis of competitive interactions in Drosophila melanogaster. Heredity, $\mathbf{5 8 , 2 7 9 - 2 8 8 . ~}$

DE WIT, C. T. 1960. On competition. Versl. Landbouwk. Onderz. Ned., 66, 1-82.

GILLESPIE, J. H. AND TURELLI, M. 1989. Genotype-environment interactions and the maintenance of polygenic variation. Genetics, 121, 129-138.

GRIMALDI, D. AND JAENIKE, J. 1984. Competition in natural populations of mycophagous Drosophila. Ecology, 65, 1113-1120.

LEWONTIN, R. C. 1965. Selection for colonizing ability. In: Baker, H. G. and Stebblins, G. L. (eds) The Genetics of Colonizing Species, pp. 79-94. Academic Press, New York.

MARKow, T. A. 1988. Reproductive behavior of Drosophila melanogaster and $D$. nigrospiracula in the field and in the laboratory. J. Comp. Psychol., 102, 169-173.

MATHER, K. AND CAligari, P. D. S. 1981. Competitive interactions in Drosophila melanogaster. II. Measurement of competition. Heredity, 46, 239-254.

MILLER, R. S. 1964. Larval competition in Drosophila melanogaster and D. simulans. Ecology, 45, 132-148.

PARTRIDGE, L., EWING, A. AND CHANDLER, A. 1987a. Male size and mating success in Drosophila melanogaster. The roles of male and female behaviour. Anim. Behav., 35, 555-562.

PARTRIDGE, L. AND FARQUHAR, M. 1981. Sexual activity reduces lifespan of male fruitflies. Nature, 294, 580-582.

PARTRIDGE, L. AND FARQUHAR, M. 1983. Lifetime mating success of male fruitflies (Drosophila melanogaster) is related to their size. Anim. Behav., 31, 871-877.

PARTRIDGE, L. AND FOWLER, K. 1993. Responses and correlated responses to artificial selection on thorax length in Drosophila melanogaster. Evolution, 47, 213-226.

PARTRIDGE, L., HOFFMAN, A. AND JONES, J. S. 1987b. Male size and mating success in Drosophila melanogaster and Droso- phila pseudoobscura under field conditions. Anim. Behav., 35, 468-476.

PROUT, T. AND BARKER, J. S. F. 1989. Ecological aspects of the heritability of body size in Drosophila buzzatii. Genetics, 123, 803-813.

RISKA, B., PROUT, T. AND TURELLI, M. 1989. Laboratory estimates of heritabilities and genetic correlations in nature. Genetics, 123, 865-871.

ROBERTSON, F. W. 1957. Studies in quantitative heritance. XI. Genetic and environment correlation between body size and egg production in Drosophila melanogaster. J. Genet., $\mathbf{5 5}, 428-443$.

ROBERTSON, F. W. 1963. The ecological genetics of growth in Drosophila. 6. The genetic correlation between the duration of the larval period and body size in relation to larval diet. Genet. Res., 4, 74-92.

RUIZ, A., SANTOS, M., BARBADILlA, A., QUEZADA-DIAZ, J. E., HASSON, E. AND FONTDEVILA, A. 1991. Genetic variance for body size in a natural population of Drosophila buzzatii. Genetics, 128, 739-750.

SANG, J. H. 1949. The ecological determinants of population growth in a Drosophila culture. III. Larval and pupal survival. Physiol. Zool., 22, 183-202.

SANTOS, M., FOWLER, K. AND PARTRIDGE, L. 1992a. On the use of tester stocks to predict the competitive ability of genotypes. Heredity, 69, 489-495.

SANTOS, M., RUIZ, A., BARBADILLA, A., QUEZADA-DIAZ, J. E., HASSON, E. AND FONTDEVILA, A. 1988. The evolutionary history of Drosophila buzzatii. XIV. Larger flies mate more often in nature. Heredity, 61, 255-262.

SANTOS, M., RUIZ, A., QUEZADA-DIAZ, J. E., BARBADILLA, A. AND FONTDEVILA, A. 1992b. The evolutionary history of Drosphila buzzatii. XX. Positive phenotypic covariance between field adult fitness components and body size. $J$. Evol. Biol., 5, 403-422.

SEWELL, D., BURNET, B. AND CONNOLLY, K. 1975. Genetic analysis of larval feeding behaviour in Drosophila melanogaster. Genet. Res., 24, 163-173.

TANTAWAY, A. O. AND RAKHA, F. A. 1964. Studies on natural populations of Drosophila. IV. Genetic variances of and correlations between four characters in $D$. melanogaster and $D$. simulans. Genetics, 65, 121-132.

TANTAWAY, A. O. AND VETUKHIV, M. O. 1960. Effects of size on fecundity, longevity and viability in populations of Drosophila pseudoobscura. Am. Nat., 94, 395-403.

TAYLOR, C. E. AND KEKIC, v. 1988. Sexual selection in a natural population of Drosophila melanogaster. Evolution, 42, 197-199.

WEISBROT, D. R. 1966. Genotypic interaction among competing strains and species of Drosophila. Genetics, 53, 427-435.

WILKıNSON, G. S. 1987. Equilibrium analysis of sexual selection in Drosophila melanogaster. Evolution, 41, 11-21.

WILKINSON, G. S., FOWLER, K. AND PARTRIDGE, L. 1990. Resistance of genetic correlation structure to directional selection in Drosophila melanogaster. Evolution, 44, 1990-2003. 